\title{
Flight Team Development in Support of LCROSS - a Class D Mission
}

\author{
Paul D. Tompkins ${ }^{*}$ \\ Stinger Ghaffarian Technologies, Moffett Field, CA 94035 \\ Rusty Hunt ${ }^{\dagger}$, John Bresina ${ }^{\ddagger}$, Ken Galal ${ }^{\S}$, Mark Shirley ${ }^{\natural}$ \\ NASA Ames Research Center, Moffett Field, CA 90435 \\ James Munger \\ Northrop Grumman Advanced Systems, Redondo Beach, CA \\ Scott Sawyer ${ }^{* *}$ \\ Orbital Network Engineering, Cupertino, CA
}

\begin{abstract}
The LCROSS (Lunar Crater Observation and Sensing Satellite) project presented a number of challenges to the preparation for mission operations. A class D mission under NASA's risk tolerance scale, LCROSS was governed by a $\$ 79$ million cost cap and a 29 month schedule from "authority to proceed" to flight readiness. LCROSS was NASA Ames Research Center's flagship mission in its return to spacecraft flight operations after many years of pursuing other strategic goals. As such, ARC needed to restore and update its mission support infrastructure, and in parallel, the LCROSS project had to newly define operational practices and to select and train a flight team combining experienced operators and staff from other arenas of ARC research. This paper describes the LCROSS flight team development process, which deeply involved team members in spacecraft and ground system design, implementation and test; leveraged collaborations with strategic partners; and conducted extensive testing and rehearsals that scaled in realism and complexity in coordination with ground system and spacecraft development. As a testament to the approach, LCROSS successfully met its full mission objectives, despite many in-flight challenges, with its impact on the lunar south pole on October 9, 2009.
\end{abstract}

\section{Nomenclature}

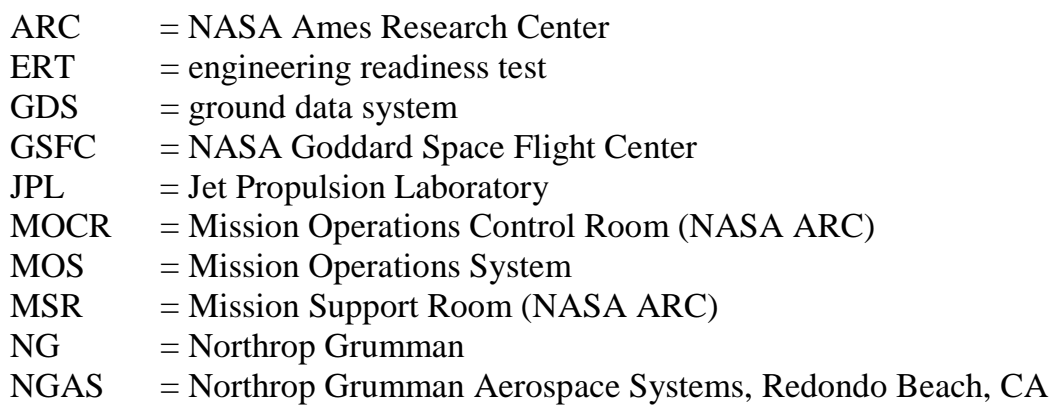

\footnotetext{
* LCROSS Flight Team Lead, Code TI, Intelligent Systems Division, M/S 269-3

${ }^{\dagger}$ LCROSS Ground Data Systems Lead, Code TI, Intelligent Systems Division, M/S 269-3

${ }^{\ddagger}$ LCROSS Activity Planning \& Sequencing Lead, Code TI, Intelligent Systems Division, M/S 269-2

$\S$ LCROSS Mission \& Maneuver Planning Lead, Code PX, Project Management Division, M/S 240-2

" LCROSS Payload Engineer, Code TI, Intelligent Systems Division, M/S 269-2

\# LCROSS NG Mission Operations Lead,

** LCROSS Mission Operations System Test Conductor, 20863 Stevens Creek Blvd, Suite 100
} 
NGTS = Northrop Grumman Technical Services, Lanham, MD

ORT = operational readiness test

ROC = Remote Operations Center

SOC = Science Operations Center (NASA ARC)

TT $\quad=$ thread test

\section{Introduction}

The preparation for LCROSS mission operations was particularly challenging due to the low LCROSS cost cap, a fast-paced development schedule, the unique and non-repeating nature of the mission, and the number of years that had passed since NASA ARC had flown a similar mission. After years pursuing strategic priorities outside the flight operations realm, NASA ARC had to undertake a significant restoration of ground data systems (GDS) and infrastructure. Meanwhile, LCROSS could not draw its mission operations team from a standing cadre of recentlyexercised ARC mission operations staff.

Despite these challenges, NASA ARC also entered with a number of advantages, namely its prior flight operations heritage and experience, collected via earlier missions and operations support conducted at NASA ARC and outside the Center; its strong staff covering a diverse set of disciplines, including spacecraft engineering, wind tunnel operations, computer science and software engineering; and, without a pre-existing culture of large-scale mission operations, the institutional freedom to tailor an operations philosophy to the lean LCROSS project model.

The remaining subsections within in Section I summarize the conditions under which LCROSS team development began. Section II describes how team development advanced in parallel with GDS and spacecraft development, and Sections III through VI detail the various means our team used to develop and tailor operational practices to LCROSS, and to train for flight. Then, Section VII provides a brief overview of LCROSS flight experience and team performance, from the human perspective, as a complement to descriptions of our experience with the spacecraft contained in Ref TBD and TBD. We evaluate our development approach, and consider its value for future missions in Section VIII.

\section{A. LCROSS Project}

LCROSS was conceived as an economical approach to determining the nature of hydrogen observed in permanently shadowed craters at the lunar poles. LCROSS was selected in April 2006,

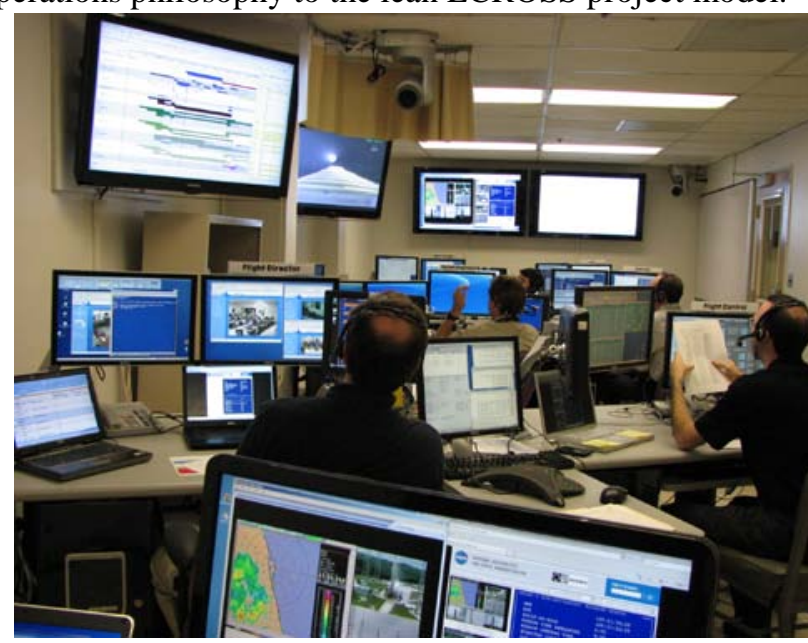

Figure 1. Flight Team in the Mission Operations Control Room (MOCR). The MOCR seated seven operators and was the focal point for execution and monitoring operations during the mission. under the Lunar Precursor Robotic Program (LPRP) of NASA's Exploration Systems Mission Directorate (ESMD), as a secondary payload to be launched with the Lunar Reconnaissance Orbiter (LRO). Under the earliest possible launch date of October 28, 2008, LCROSS was obligated to meet a 29 month development schedule from ATP through launch readiness ${ }^{\dagger \dagger}$. The LCROSS project was constrained to a \$79 million cost cap, of which \$TBD million was designated for mission operations. The project's tight budget and fast-paced schedule provided a strong impetus for utilizing a small staff in creative ways to accomplish the huge body of work required to prepare for flight.

To ease the budget and schedule challenges, NASA designated LCROSS as "Class D" under its risk tolerance scale. This classification allowed the project to accept greater risk relative to higher-profile, large-budget missions, and shaped the development strategy and operational approach. However, the definition of Class D was not welldocumented, nor did it have a strong precedence in prior NASA missions. Hence, the LCROSS project team had to work out how the classification translated into actual programmatic, engineering and operational practice, in coordination with (and often with the approval of) NASA ARC, the program office and NASA headquarters.

\footnotetext{
I† Though the LRO/LCROSS launch date ultimately slipped to June 18, 2009, LCROSS met the original deadline for flight readiness in October 2008.
} 
Importantly, it had to balance the need to reduce cost and save time against the natural tendency to pursue the reliability standards of bigger-budget missions.

\section{B. Initial State of Mission Operations}

The LCROSS mission operations campaign was the first NASA ARC had led since the Lunar Prospector (LP) mission, which ended in $1999^{1}$. In the intervening years between LP and LCROSS, while ARC pursued a number of other high-priority strategic goals for NASA, the ARC mission operations infrastructure was specialized for other mission domains, including Space Shuttle payload operations support, and in other cases was not actively maintained. NASA ARC was concurrently preparing for the Kepler mission ${ }^{2}$. However, spacecraft operations for that mission were to be conducted outside NASA ARC, and science operations were being performed in a separate NASA ARC facility, and followed a very different operational model.

Another consequence of the many years between flight missions at ARC was the minimal number of available, recently-exercised operations staff from which an LCROSS team could be selected. Though some team members had flight operations experience, many came from other backgrounds and were transformed into experienced team members through LCROSS.

Without a continuous history of space operations at ARC, and without an active set of practices that are typically carried from one team to the next in an active mission operations facility, the LCROSS team also needed to define or borrow the full range of generic operational practices. In retrospect, this provided both a disadvantage and an advantage. In one respect, this added a large body of work to the team beyond LCROSS-specific preparations. However, the absence of a pre-defined set of practices allowed the team to scale its process to the needs and constraints of LCROSS.

\section{Team Development Process}

With little operational culture to drawn from early in the LCROSS project, the Mission Operations System (MOS) team had to define everything about how it would operate. Broadly, the MOS needed to define its composition and its body of operational practices, both general (e.g. voice loop protocols, Deep Space Network interaction, telemetry data archival, anomaly resolution processes) and LCROSS-specific (including the mission plan, team roles and responsibilities, team interactions and data transfers, command product generation and verification, procedures, flight rules, etc). Through a team training and test plan, which it also needed to invent, it would prepare its operators for flight.

On the selection of LCROSS, NASA ARC initiated a mission operations facilities and ground systems restoration effort that continued in parallel with LCROSS spacecraft and mission operations team development. Hence, the team, spacecraft, and ground system grew together. The MOS was responsible for defining requirements for mission operations facilities and the GDS, whose implementation was handled under project-external ARC funding. Details of the ground systems development are described in Hunt et al. (Ref TBD).

LCROSS adopted an iterative approach to both MOS and GDS development, recognizing that without key elements in place and lacking realism in early tests and simulations, operational practices and even team composition might require modification. Training occurred on a gradually-developing ground system target, sometimes with serious limitations in capability, until late in the development schedule when all elements were in place. MOS process definitions were refined gradually through collaboration and repeated simulations.

Of course LCROSS relied heavily on the experiences of earlier mission operations teams. It selected from previously-successful operational practices wherever possible, as long as they were relevant to the LCROSS mission and fit within the lean LCROSS model. Given the team's experience mix, it took specific inspiration from Lunar Prospector, the Mars Exploration Rover (MER) mission, Space Shuttle and International Space Station (ISS) operations, commercial communications satellite operations, prior Northrop Grumman missions, and even from ARC wind tunnel operational practices.

\section{Composing the Team}

The LCROSS mission plan was characterized by two focused periods of intense operations separated by months of lower-intensity spacecraft monitoring and occasional critical events. The MOS team structure reflected the need to efficiently support both high- and low-intensity operations. The MOS was built on a light weight core team at NASA ARC, supplemented with key maneuver design and navigation expertise from JPL and GSFC that could support most operational roles for the mission. For the most intense periods and other critical events, the MOS also included an extended team of spacecraft engineers from NG to provide real-time, deep expertise in the behavior of spacecraft systems. 


\section{A. The Core Team at ARC}

As a starting point, the LCROSS MOS team adopted a team structure and roles typical of other orbiting science missions (see Figure TBD). However, LCROSS budget limitations dictated a small and efficient MOS staff. LCROSS selected a core team of individuals to fill the roles of each MOS subsystem, but in most cases the staff count was barely enough to fill the needs of the role (1-2 people each subsystem), and did not allow for a backup operator. In some cases, given a particular mix of skill and experience, staff members were assigned to more than one role. Furthermore, LCROSS could not afford the luxury of employing fully-independent operations and GDS development teams. In fact, many operators on the team began work on LCROSS as GDS developers and later inherited additional responsibilities as mission operators. Importantly, early in LCROSS development, we recognized the benefit of including members of the science and payload engineering teams in operations. The role of Payload Engineer was added to the MOS, and was represented by a console position (one of seven) in the Mission Operations Control Room (MOCR).

Prior to joining LCROSS, NASA ARC flight team members came from a diverse set of backgrounds. Some served in other mission operations roles, including in support of Lunar Prospector, Gravity Probe B, and other orbital science missions; the Mars Exploration Rover mission; Space Shuttle and International Space Station operations; and commercial satellite missions. Others were employed as engineers in support of space and defenserelated engineering projects. A number of staff once led or supported wind tunnel operations at NASA ARC, and many had been researchers or software developers in the fields of autonomous systems, artificial intelligence and robotics. Regardless of background, all team members started as technically very competent in their respective fields, and all were very excited and motivated by the prospect of supporting operations for a lunar impact mission.

As we describe later, concurrent with operations training, LCROSS operators served in a wide range of design, development and test roles both for the ground system and the spacecraft itself. Despite the extra work and split assignments, that experience was advantageous, and highly relevant to operations.

\section{B. External Team Members}

Beyond the small core team of MOS developer/operators at NASA ARC, the LCROSS team partnered with other organizations to bolster ARC experience in key areas, to create a reserve capacity for key mission phases, and to support spacecraft anomalies, should they occur.

Due to the particular challenges of LCROSS trajectory planning and navigation for precise lunar impact targeting, NASA ARC augmented its own expertise in these areas with partners from the Jet Propulsion Laboratory (JPL) (providing navigation and orbit propagation expertise) and NASA Goddard Space Flight Center (GSFC) (providing complementary trajectory and maneuver planning capabilities). JPL also assigned a small team to LCROSS devoted to Deep Space Network (DSN) communications link scheduling.

The MOS also needed to possess deep spacecraft systems and subsystems expertise to assess spacecraft performance throughout the mission, and to diagnose and suggest remedies for anomalous spacecraft behavior, as necessary. As a small project, LCROSS employed only two ARC spacecraft engineers - the Project Systems Engineer and his deputy - to oversee spacecraft development at Northrop Grumman. Both were ultimately assigned as Systems Engineers on the flight team. However, with only two ARC LCROSS spacecraft engineers to call on, and lacking the time to independently train a new set of ARC staff in the detailed design of LCROSS hardware and software, a natural approach was to augment the MOS with the team of Northrop Grumman spacecraft systems and subsystem engineers who were serving as LCROSS lead spacecraft designers and integration and test engineers (see Figure TBD). There were no better candidates in terms of depth of knowledge, and many were excited at the prospect of participating in operations. Two Northrop Grumman units were involved in LCROSS development: Aerospace Systems in Redondo Beach, CA (NGAS), and Technical Services in Lanham, MD (NGTS). Engineers were drawn from both into the MOS.

Despite the clear benefits, this approach presented a number of additional challenges:

1) LCROSS would have to devote budgetary resources to train these engineers (through classes, tests and rehearsals, etc), many of whom were neither experienced in operations generally, nor in the LCROSS concept of operations specifically.

2) Because these engineers also served in leading roles on LCROSS spacecraft development, they were unlikely to be able to support all operational training activities (concurrent with spacecraft testing).

3) The spacecraft engineering team needed facilities and equipment to allow them to interact in real time with telemetry. To co-locate them at ARC would entail a substantial increase in operational floor space, an enormous travel budget, and a significant travel burden on each of the participants (with high attrition likely). A distributed solution would require the build-up of dedicated remote operations rooms at each of 
the Northrop Grumman facilities, and would introduce the difficulties of distributed team coordination during rehearsals and flight.

4) The LCROSS project could not afford to employ the full set of spacecraft engineers full-time for the entire flight phase. Part-time involvement required that engineers work on other projects. During flight, without management support and careful staff planning, these engineers were at risk of being fully claimed by other Northrop Grumman projects.

\section{Team Collaboration during Development and Test Phases}

The core MOS team comprised the members at ARC, the Navigation Lead at JPL, and two Northrop Grumman systems engineers. The ARC team shared some management responsibility with lead members at JPL and Northrop Grumman for coordinating their teammates.

A key to MOS success was its tight integration and culture of open communications across organizations. Small team size enhanced the MOS's ability to communicate and to remain focused and coordinated. Most MOS development and coordination meetings were open to the entire "core" team, and often to the broader team.

All organizations demonstrated full commitment to the LCROSS project. Of critical importance, management at all participating organizations successfully established an atmosphere of collaboration, without barriers. Furthermore, inter-organizational relationships were not strictly bounded by standard contract limitations. Northrop Grumman openly voiced problems encountered during spacecraft development, and involved ARC at every stage in working through these obstacles. As full-fledged MOS team members, Northrop Grumman engineers regularly reviewed and steered the development of spacecraft operational procedures at ARC and, through participation in procedure development and training exercises, they could also better understand the operational effects of remaining spacecraft design decisions. As described in Section VI, ARC MOS engineers were also included in key aspects of spacecraft development and test, further solidifying this collaborative bond. Finally, tight cross-organizational team integration allowed external participants to communicate to each other effectively. For example, the JPL Navigation team worked closely with Northrop Grumman attitude control engineers to develop models of the perturbation effects of various controller modes on the trajectory. Through this body of interactions, and because of the tone set by management early in the project, the MOS (and the project at large) became a cohesive unit concerned principally with mission success, despite political and organizational boundaries.

In balance, baselining a distributed MOS presented other challenges. Though the team was well-integrated across organizational lines, communications breakdowns and isolation sometimes occurred due to physical separation. With the bulk of the team at ARC, informal discussions there occasionally unintentionally excluded external partners. Even full-team teleconferences were prone to poor audio quality and acoustics or occasionally poor network or graphics-sharing software performance. The importance of effective distributed team coordination was recognized early in the MOS development, and was reflected both in MOS operational processes and in the GDS design (voice loops, video sharing, improved teleconferencing equipment, etc.).

\section{Development of Operational Practices}

LCROSS operational practices were built on standard models of space operations, tailored to the LCROSS mission plan (see Ref TBD for a description of flight operations). The most fundamental of these practices are expressed in the choice of team organizational roles, described in the previous section. How these team members operated together is the subject of this section. The full set of practices is too large to describe in detail here, but the following sections highlight some of the salient features of LCROSS practice.

\section{A. Workflow and Shift Scheduling}

LCROSS operated under a typical workflow model with four phases: Planning, Command Generation and Verification, Execution, and Assessment. Because the LCROSS mission was largely absent of routine operations, the workflow strongly emphasized the first two phases. Because LCROSS operated at close range to Earth, Execution and much of Assessment could be conducted in near real-time. A full cycle, depending on the operation, lasted anywhere from 7 hours to two days:

1) Planning: During this phase, the Mission and Maneuver Design team designed trajectory correction maneuvers and spacecraft attitude changes to meet the requirements of impact targeting, science instrument calibrations, communications, and other activities, while minimizing propellant consumption. Payload Engineers and Science Team members designed specific payload observation sequences, as applicable, and Systems Engineers designated housekeeping activities to be executed. Link Schedulers coordinated Deep Space Network contact periods for LCROSS, and Link Analysts predicted link performance for future 
contacts based on past experience. Planning phase culminated in an Activity Selection Review, during which the team reviewed maneuver designs and selected and ordered the supporting activities to be undertaken during a future contact. The resulting fully-ordered list of activities and their associated parameters was called an Activity Plan. The Mission and Maneuver Design Engineers operated in multiple overlapping shifts during peak activity periods; all others were individuals operating in a single shift.

2) Command Generation and Verification: During this phase, the Command Sequencing Engineer converted the Activity Plan into a Command Plan, combining onboard command sequences and ground-based commanding procedures. This process was partially automated to minimize human error, but was sufficiently flexible to enable in-flight modifications. The team verified the correctness and safety of the command products using a combination of analysis (e.g. rudimentary automated flight rule checking) and simulation. The LCROSS simulator combined ground copies of spacecraft avionics, ran spacecraft flight software, and simulated spacecraft dynamic behavior. This phase concluded with the Command Approval Meeting, during which the team reviewed all Command Plan elements and associated analysis and simulation results before providing final approval to proceed. The minimal team supporting Command Generation and Verification comprised a single shift of activity.

3) Execution: In this phase, the MOS enacted the Command Plan. All execution was conducted by the team in near real-time (with speed-of-light delays of seconds) and typically resulted in the collection of all associated telemetry for concurrent or subsequent review. Execution was closely orchestrated by a Flight Director, and all commands were sent exclusively by a Flight Controller. During periods of 24-hour contact with the spacecraft, the Execution team was divided into two shifts, A and B, each staffed with operators for every core role. Often one shift actively monitored the spacecraft during Planning and Command Generation phases, and the other performed Execution. During periods with sporadic spacecraft contacts, single Execution shifts conducted the operations for each given DSN pass. Science team operators augmented the standard Execution team for events focused on science data collection.

4) Assessment: This phase ran in parallel with Execution phase and persisted while the team estimated the trajectory, characterized orbit perturbations and evaluated burn performance (Navigation), analyzed engineering telemetry (spacecraft and payload engineers), and analyzed science data (science team) to infer spacecraft health status and to determine the degree of success for maneuvers and science activities. This information was fed back into the next Planning phase, and to occasional status briefings for project stakeholders.

Day-to-day operations were led by the Flight Director on duty. The Mission Operations Manager ensured that the flight team adhered to accepted operational practices, helped coordinate during anomaly responses, and was the primary communicator between the flight team and project management and LCROSS stakeholders (NASA ARC, LPRP program, and NASA headquarters).

\section{B. Facilities, Physical Distribution and Team Communications}

The organization of LCROSS facilities was strongly influenced by the workflow design and the mix of internal and external MOS team members. Operations were centered at ARC, and utilized three primary rooms, all of which were built up and equipped in stages during LCROSS development (for technical details on facility and GDS design, please consult Ref TBD). Mission Operations Control Room (MOCR) activities focused on the execution of spacecraft commands and spacecraft monitoring (Execution and Assessment Phases). The MOCR was a selfcontained facility that accommodated a core set of seven operators with telemetry and command workstations and primary and backup telemetry and commanding data systems. The Science Operations Center (SOC) was the room from which the Science Team evaluated science instrument data streaming in real-time from the spacecraft during science events, coordinated the ground telescope observation campaign at lunar impact, and performed some of the post-event science data analysis. The SOC was separated physically from the MOCR to permit science-oriented discussions and parallel activities without disturbance to the MOCR team. The Mission Support Room (MSR) was the focus of activity during the Planning and Command Generation and Verification Phases, and also portions of Assessment Phase. It was designed as a center for off-line trajectory planning, development and test of command products, and discussion of engineering issues (including anomaly resolution). The MSR was separated from the MOCR and SOC to allow discussions and coordination in parallel with real-time and science activities.

Outside of ARC, Northrop Grumman assembled and maintained two Remote Operations Centers (ROCs), one at the NGAS facility in California, and another at NGTS in Maryland. These supported two roles. First, the NG ROC's acted as an extension of the MOCR, providing real-time oversight of subsystem and system behavior during Execution phases. Second, they acted as engineering extensions of the MSR for more detailed review and discussion of spacecraft behavior (both nominal and anomalous), with easy access to NG design and test repositories 
and factory support (Assessment). Most of the subsystem engineering disciplines and the Systems Engineers operated from NGAS, while the subsystems engineers overseeing Avionics and Flight Software operated from NGTS. The JPL Navigation and Link Scheduling teams operated from their own fully-equipped facilities, while GSFC Mission Design team partners supported the critical initial and final weeks of the mission from the MSR at ARC, but worked from their home facilities during the months of Cruise Phase.

A major challenge was enabling effective communications and situational awareness over this distributed team. We discovered in early tests that standard voice communications, on their own, were insufficient to coordinate the team and to keep operators informed on minute-by-minute progress during Execution or in reviewing planning and command generation results. Achieving good communications and situational awareness became a major effort in LCROSS GDS development (TBD ref here). Many of these elements were introduced gradually into operations over the LCROSS buildup.

The primary means of communications for Execution phase remained a voice loop system that linked all operations consoles as well as operators at the launch site and the Deep Space Network. Multiple channels allowed the team to compartmentalize communications according to purpose and criticality. For Activity Selection Reviews and Command Approval Meetings (see Section TBD), the team collaborated via conference telephones and a secure meeting system that allowed

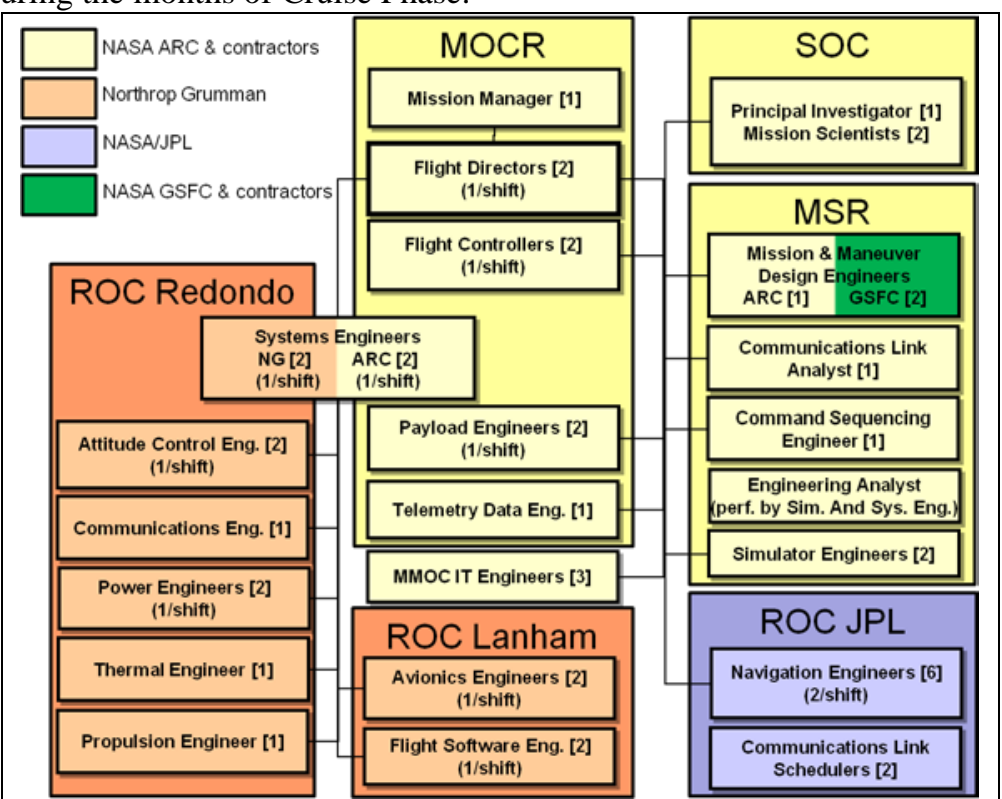

Figure 2. LCROSS Flight Team and Physical Distribution. Small boxes represent the full set of flight team staff, while encompassing boxes represent facilities from which they most often worked. Planning and Command Generation \& Verification Phases were concentrated in the MSR. Execution Phase was led by the MOCR, often with assistance from Systems Engineers at NG. Critical events were also supported by subsystems engineers, up to the full set of disciplines, depending on need. The SOC and Payload Engineers participated in all science/payload activities.

network-based distribution of graphical presentations. All team workstations were networked to a Mission Data Product Server that served as the central repository and transfer point for data products.

For situational awareness, operators at all facilities were equipped with workstations to provide access to realtime telemetry. Workstations mirrored the display of the single commanding workstation in the MOCR to enable viewing of commands as they were being issued to the spacecraft. A video distribution system and large overhead monitors enabled rooms at ARC to mirror workstation displays in other ARC rooms. For real-time operations, ARC distributed a display of the current procedure step to all facilities, and also a real-time animation of the LCROSS spacecraft and trajectory that was keyed off of telemetry to depict the attitude state of the vehicle and fields-of-view of all sensors. ARC also provided software to depict the detailed LCROSS event timeline, which was synchronized with UTC to show the current activities, time since past events and time until future events.

\section{Tight integration of Science/Payload with MOS}

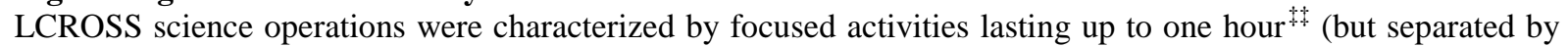
hours, days or weeks), during which the science team planned to collect image and spectroscopy data, assess instrument performance and make parameter adjustments in near real-time. Over the course of development, the MOS came to recognize that without tight coordination with the science and payload teams both prior to and during flight, it could not necessarily ensure that it would meet the needs of the science team during these events, or establish and maintain a science operations concept that was consistent with the rest of operations.

Over the development period, the MOS endeavored to make scientists and Payload Engineers integral to operations. Scientists and Payload Engineers became required signatories for the approval of any command product

\# Thermal constraints dictated that the LCROSS payload could not be powered on for more than one hour at a time. 
involving the instrument payload. For Execution Phase, the Payload Engineer was assigned one of seven consoles in the MOCR and led the assessment of payload engineering performance during all payload activities. The SOC was equipped with science workstations networked to the MOS-wide telemetry data server, enabling scientists to view and assess instrument data seconds after capture on the spacecraft. To keep real-time commanding authority consistent with other operations, science team members and the Payload Engineer made command requests (from a pre-arranged set) to the Flight Director verbally over the voice loop. The Flight Director quickly evaluated the criticality of science requests relative to other immediate ground-commanded tasks, and passed them on to the Flight Controller for radiation. This preserved a notion of centralized authority at the "big picture" level, albeit with some added delay. In cases where time was especially critical, the MOS devised more immediate responses to specific science team requests.

The science and payload teams worked closely with the MOS over many months to develop command sequences and procedures to address the full range of anticipated science activities. To test these designs and ultimately to train for flight, the same team members were integral participants in all science-oriented MOS tests and rehearsals.

\section{Operational Training and Test Program}

The LCROSS MOS training program included a combination of conventional and less conventional training methods. This section describes all of the more conventional approaches we used to ready ourselves for flight. In addition to attending class-based training, operators were encouraged to attend spacecraft design reviews through Critical Design Review, and were obligated to attend operations-oriented seminars and workshops focusing on specific spacecraft subsystems and mission events. Operator certification was not formalized, but was judged informally by peers and MOS leadership over the course of many system-wide tests and rehearsals.

\section{A. Ground Data System Training}

Most training in the use of GDS tools (telemetry and commanding software, voice loops, link analysis, etc) was performed conventionally, either through formal classes taught by software vendors, through classes taught by key MOS team members to the broader team, or informal instruction between team members followed by on-the-job practice. Many GDS tools were custom designed and implemented for LCROSS, and in some cases the developers were the end users of the software. Partial-team and system-wide tests provided the team with many opportunities to practice GDS tool usage.

\section{B. S/C Reviews as Training Opportunities}

An important part of training was to familiarize team members with the spacecraft design. The MOS recognized spacecraft design reviews as valuable training opportunities, and required its membership to attend detailed subsystem Critical Design Audits presented by Northrop Grumman. The ARC Project Systems Engineer and his deputy (also MOS Systems Engineers) interacted closely with NG throughout the spacecraft development and test, and consequently became two of the most knowledgeable MOS team members with regards to spacecraft design.

\section{Workshops/Seminars}

To complement training via spacecraft design reviews, the MOS held a series of Spacecraft Subsystem Workshops, led by an NG lead subsystem engineer (and often assigned to the MOS). The team conducted workshops for the Attitude Control, Avionics, Flight Software, Power, Propulsion, Communications, Autonomy and Fault Management, and Payload subsystems. Each workshop lasted from four to eight hours and reviewed subsystem design, but emphasized subsystem operation and upkeep, including the use of primary relevant commands and telemetry, typical on-orbit behavior, troubleshooting and operational constraints. All ARC MOS members were required to attend these workshops.

A series Operational Focus Workshops, led by the Lead Flight Director, provided in-depth reviews of operations for specific mission events at the system level. These reviewed the sequences of events, DSN utilization, preplanned command sequences, operational procedures, the workflow covering the events, timelines and staffing. Workshops were held for Activation and Checkout; Cruise Phase Housekeeping; Cruise Phase Trajectory Correction Maneuvers; Star Field Calibration and Lunar Swingby; Earth Look Calibrations; and Separation and Lunar Impact. All MOS team members were required to attend these workshops.

In conjunction with the Operational Focus Workshops, the MOS conducted many workshops to develop and review command sequences for the same events. These meetings gathered spacecraft subsystem and systems-level expertise, Flight Directors and Flight Controllers, and for science-related activities, members of the science and 
payload team to communicate design requirements to the Activity Planning \& Sequencing Lead, who implemented the sequences and, for parameterized activities, tools to be used to generate the sequences during flight.

\section{MOS Operational Tests}

With the concurrent development of the MOS, operational facilities and GDS, MOS training was limited by the degree of availability of facilities and tools, as governed by the GDS development schedule. The LCROSS team recognized two things: first, that MOS training could not wait until the facilities and GDS were fully deployed, and second, that the GDS team could not confidently deploy a quality product without intermediate validation testing by the MOS team. Hence, the MOS coordinated with the GDS development team to create a schedule of interleaved GDS releases and MOS operational tests (of procedures and processes) that also served as training exercises and GDS requirement validation tests. The GDS release schedule was designed to provide increasingly capable sets of hardware, software and command products that built logically upon previous releases, but that were usable in intermediate form to support MOS tests. MOS tests grew in sophistication and realism, starting with tests of contiguous "threads" of the operational workflow, and growing to system-wide tests closely approximating the operations for full mission events. MOS tests were designed with full knowledge of, and limited by, the capabilities of each GDS release. Similarly, the scope of GDS releases was driven in part by the needs of upcoming MOS tests.

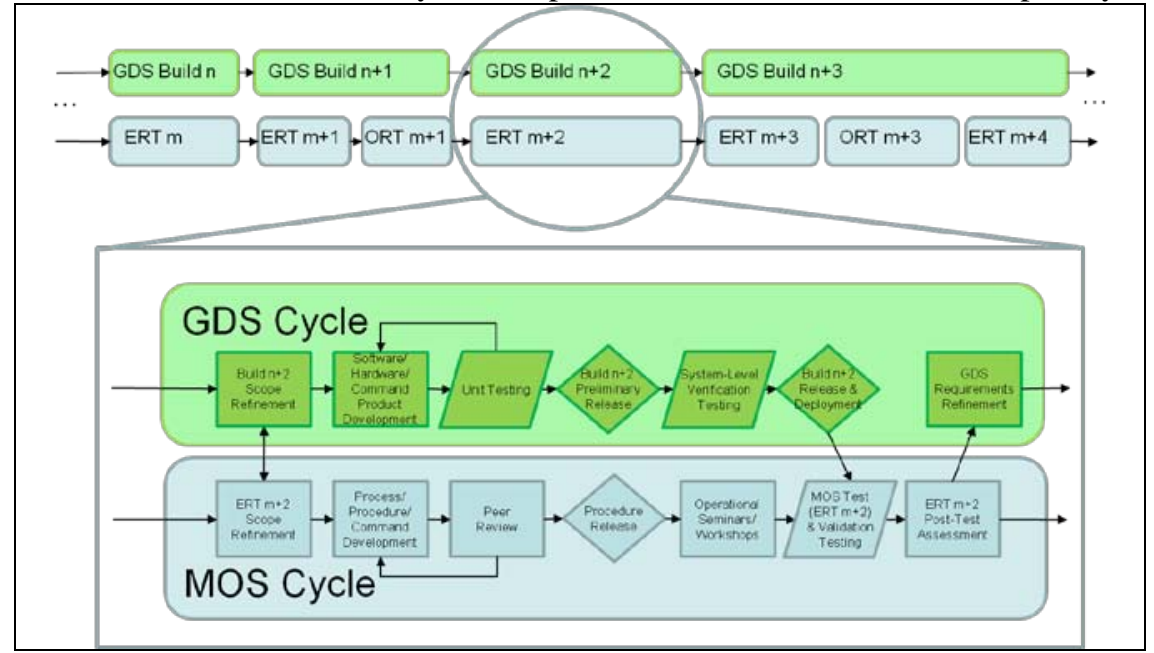

Figure 3. Interleaved GDS and MOS Development Cycles to Support Parallel Development. GDS cycles provided increasing system-wide capabilities. MOS cycles focused on specific mission events and drove development of event-specific GDS elements. Operationally-realistic MOS tests doubled as GDS requirements validation tests; results occasionally prompted the refinement of GDS requirements. In general, GDS releases served multiple MOS development cycles.
An important piece of the GDS delivery was the LCROSS spacecraft simulator. It combined a partial copy of LCROSS avionics hardware, a full copy of flight software, and a software-based dynamic simulator to simulate vehicle flight dynamics and the behavior of other spacecraft systems not represented in hardware (e.g. IRU, star tracker, power electronics and solar array, thruster modules). Importantly, the simulator was built from pieces of the simulator used by NG to develop LCROSS flight software. Therefore, MOS validation tests simulating the behavior of the spacecraft could not be conducted until the completion of main-line flight software development. (TBD: is this correct, or did NG give up pieces of their sim much earlier than FSW delivery?) However, once transitioned to the MOS, the LCROSS spacecraft simulator became a dedicated resource for GDS development (for command products) and for MOS tests (during which it represented the spacecraft). The simulator was critical to the success of preparations for LCROSS.

Early tests were designed and conducted by the MOS Flight Team Lead, who also was the Lead Flight Director. As tests became more complex, the MOS recognized that it needed a dedicated Test Conductor to design test scenarios and to steer the course of events of tests (including operation of the LCROSS spacecraft simulator) behind the scenes. The Test Conductor was selected for his extensive prior experience with mission operations and spacecraft systems engineering. Hence, after being trained in the specifics of the LCROSS mission and spacecraft and operation of the simulator, the Test Conductor could comfortably lead system-wide tests and independently judge the performance of the MOS team.

MOS tests of operational "threads" were called Thread Tests (TTs), and began with tests of telemetry receipt (telemetry decoding, distribution and display), commanding, and data product routing and archival, to name a few. Later TTs tested more significant threads, for example planning and command generation for trajectory correction maneuvers. In addition to supporting GDS validation tests, TTs helped train operators in the use of GDS tools, in 
the use of realistic basis data to create and deliver mission data products, and also to accustom them to segments of the MOS workflow.

With the availability of all ARC operations rooms and sufficient GDS readiness, the MOS introduced systemlevel Engineering Readiness Tests (ERTs) that brought a significant portion of the team together and tested the complete workflow in support of specific mission events. The primary goal of ERTs was to prove that all ground elements were ready to support specific mission events, while team readiness was secondary. Hence, ERTs allowed intermediate stops and starts, timeline stretching and compression, and event re-ordering to meet team schedule constraints and to focus on the most important aspects of the test. ERTs sometimes exercised contingency execution paths, but always warned the MOS team in advance.

Once the GDS and MOS processes were fully tested at least once for a particular event, the MOS conducted an Operational Readiness Test (ORT) for the same event. ORTs were like ERTs, except that they adhered more strictly to the mission timeline (duration and ordering), and focused on validating MOS processes and team readiness over GDS tools. ORTs also tested contingency paths, but without warning the MOS team. Being able to correctly plan, execute and assess a mission event within the constraints of the mission timeline was a key validation of tools and processes, and a strong indication of operational readiness.

Rehearsals were conducted as final training events before launch and also during flight. Unlike ORTs, rehearsals typically exercised two or more days of continuous operation (active and inactive periods), mimicking a segment of the mission timeline to the minute. The Test Conductor ran rehearsals with the highest possible fidelity, and the team was expected to exercise every MOS process exactly how it would in flight. Rehearsals also exercised ancillary support activities like planning catered meals for the team during 24-hour operations, and securing and using on-site lodging for any operator living beyond a maximum range to avoid the dangers of driving after long shifts. The MOS conducted a First Week Rehearsal prior to launch covering launch through Lunar Swingby (six days in duration), and a Last Two Days rehearsal during flight covering the final trajectory correction maneuver, Centaur separation and lunar impact (two days in duration). A significant result from these tests is that team members grew to fully appreciate the rigors of the mission timeline, and discovered their own strengths and weaknesses in adapting to unusual sleep and waking hours while supporting critical events. Furthermore, with all team members involved in around-the-clock operations, with shifts on opposing sleep schedules, team members came to understand the importance of good inter-shift communications, when the full team was available. (TBD: other rehearsals? I've already forgotten!)

Special tests: Flatsat and End-to-End Testing

\section{Training Through Development and Test}

In addition to the more conventional training approaches, many of the MOS team enhanced their training through participation in spacecraft, payload and GDS development and testing. Many of these assignments were set up explicitly as training engagements, while in other cases, staff started as developers for the project, and evolved into operators. In the active design, test and review of the spacecraft and GDS, operators became far better acquainted with mission systems than would have been possible by classes and rehearsals alone. Dual responsibilities also had their share of disadvantages.

\section{A. MOS Team in Spacecraft Development}

As discussed earlier, the ARC Project Systems Engineer (PSE) and Deputy PSE were both assigned as lead Systems Engineers on the MOS. Furthermore, the MOS enlisted many NG LCROSS spacecraft engineers to augment the core ARC team. Many of these NG staff members held lead subsystems or systems design roles, or were lead integration and test engineers. Essentially, the MOS benefitted from the inherent training each of these engineers acquired over more than two years of deep involvement with LCROSS design, construction and test. Maintaining the engineering team through the entire flight saved the project significantly in training time and coordination effort. The MOS trained these engineers in operational practices (in the same training pipeline as for the rest of the MOS) far more easily than it could have trained general spacecraft engineers in the intricacies of LCROSS systems design and test behaviors (given the much greater body of knowledge, but without a training program in place).

\section{B. MOS Team in LCROSS/LRO Testing}

Under the theory that MOS team members could not possibly build a sufficiently deep understanding of LCROSS spacecraft designs and operation by attending reviews and studying documentation, the LCROSS project negotiated with Northrop Grumman to embed two team members into the spacecraft test flow. One served on-site at 
NASA GSFC as a technical liaison between the LRO and LCROSS projects, focusing on common hardware development and test. He also served at NGTS as an avionics and flight software test engineer during avionics unit testing and integrated "flatsat" testing. The second served on-site at NGAS as a liaison and interface between NASA ARC and NG systems engineers for science payload integration, the S/C build and S/C integration and test (I\&T). He also served as an interface between the I\&T team, split between NG and the launch facility, and the MOS at NASA ARC during the conductance of S/C end-to-end testing.

As a result of their assignments, these operators became two of the most knowledgeable on the team in the detailed operation of the spacecraft. Both were assigned as primary Flight Controllers, the operators directly responsible for sending commands to, and acquiring telemetry from, the spacecraft. However, during their remote assignment over months during MOS development, the remainder of the team could not regularly call on their expertise and support of overflow work. Furthermore, these volunteers spent 11 and 16 months, respectively, displaced from home.

\section{MOS Team Members as Developers of Onboard Command Sequences}

In another collaboration between the MOS and Northrop Grumman spacecraft development, the team member designated as Command Sequencing Engineer (the operator in charge of implementing MOS command sequences before and during

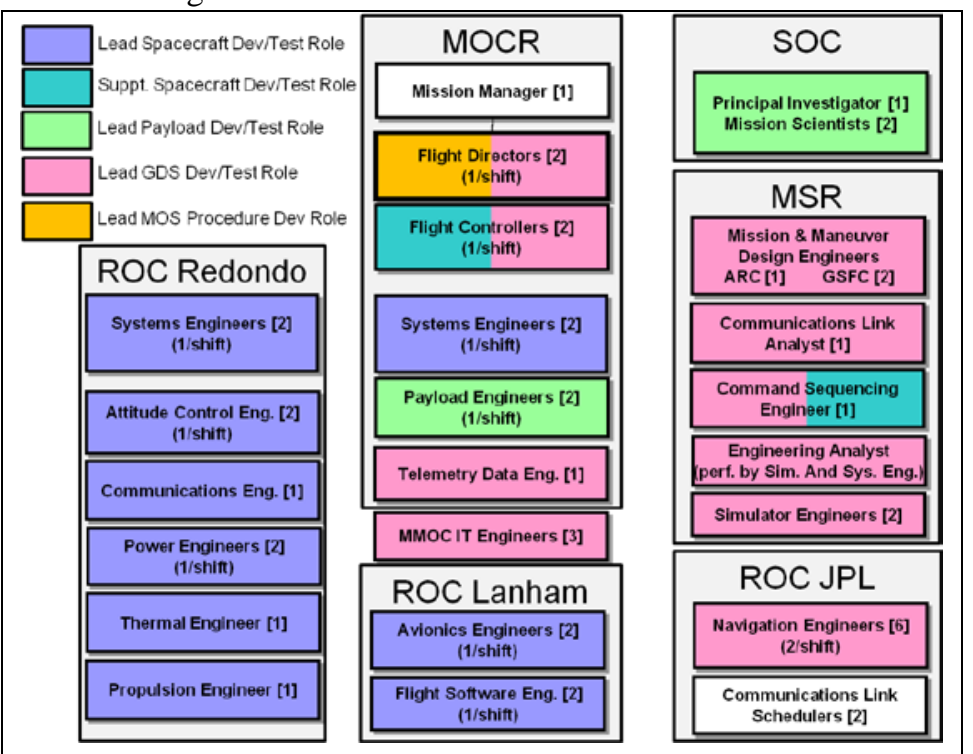

Figure 4. MOS Operator Roles in Development. Most MOS staff played key roles in spacecraft, payload, GDS, or MOS procedure development. This simplified training, but contributed to a heavy workload and occasionally hindered simultaneous MOS and GDS development.

flight) was assigned the responsibility of implementing the command sequences used by the spacecraft autonomy and fault management (A\&FM) system for critical events, including initial spacecraft power-up and onboard fault responses. Working closely with Northrop Grumman systems engineers who designed the command sequences, this team member became expert with using the command and telemetry databases, onboard command sequence authoring and compilation, and the basic operation of onboard subsystems.

\section{MOS Team in LCROSS Payload Development}

The Payload Flight Software Lead for the science payload evolved naturally into the primary Payload Engineer for flight. In his development role, this team member designed and implemented all supplementary flight code for the operation of the payload, implemented all instrument command sequences for specific science activities, and also designed and implemented software used by the science team to analyze imagery and to assess payload throughput. He also supported payload testing. No other experience could have prepared this person so thoroughly for the position of Payload Engineer.

One of the science team members also served as Payload Test Engineer during payload development, and took a key role in science operations from the SOC during flight. This test experience complemented her role as a mission scientist, and enabled her to provide additional oversight of payload performance during science activities.

The only negative aspect of this arrangement was that when the timing of MOS test exercises conflicted with payload development and test activities, these two operators generally had to prioritize their payload responsibilities.

\section{E. MOS Team in GDS Development}

Several MOS team members either oversaw GDS development or developed software tools for their respective MOS subsystems. The GDS Lead, in charge of all LCROSS ground system development, became one of two Flight Directors. While larger teams might benefit from having dedicated software developers, the LCROSS MOS found distinct advantages in having its software end users also develop code. Most importantly, the problem of accurately communicating detailed requirements is obviated when the customer and developer is the same person. This also goes for software training - the software developer is the most familiar with a tool's capabilities, limitations and 
idiosyncrasies. This is especially important in flight, when deep software knowledge could enable an operator to work around a bug that might otherwise interfere with the support of an important mission event. Other MOS team members served as GDS team members, but in capacities distinct from their operational roles, with little training advantage.

Despite these advantages, having dually-tasked operators had its share of disadvantages. For one, when developer and operator are distinct individuals, the developer can perform unit-level testing, then pass the software to the operator to perform independent requirements verification testing. When one person serves in both roles, another operator, often either less well-trained, or even from a different discipline, must perform independent verification testing. Furthermore, peak pre-launch workloads often volleyed between the GDS and MOS teams according to the interleaved GDS and MOS test cycles. For those working on both teams, the workload was extremely difficult and afforded them little rest time. Also, because MOS and GDS work often needed to happen concurrently (e.g. software development and MOS rehearsal preparations), one task or the other often suffered.

These disadvantages continued in flight. For a number of reasons, LCROSS operations were on average more busy than anticipated. Inevitable GDS bug fixes and enhancements were developed during flight, but developer/operators were too consumed with flight duties to perform global GDS deployments. The team had to resort to less-formal point deployments for specific tools, complicating the GDS configuration management task.

\section{Summary of Performance in Flight}

Arguably, the best measure of flight team preparation is its level of performance during its flight mission. This section provides a brief synopsis of LCROSS MOS performance during 112 days of flight, from a human standpoint.

Transfer Phase was six days of 24-hour operations, covering launch through lunar swingby, and the most challenging nominal segment of flight, save the pre-impact sequence. The MOS operated in two overlapping shifts of 13 hours, synchronized with major events. The MOS successfully performed all nominal events, and simultaneously responded to several in-flight spacecraft anomalies that forced some significant operational changes from the baseline to maintain spacecraft health. On Day 3, a combination of a lack of situational awareness (stemming from one of the anomalies) and operator error caused the spacecraft to transition to a "safe mode", with no lasting mission effect or major influence on other events. By the end of Transfer Phase, the team was noticeably fatigued, particularly Shift B which had worked graveyard hours.

Cruise Phase, the bulk of time in flight, was more difficult than anticipated. With anomalies from Transfer Phase still present, the early part of Cruise was spent developing sustainable workarounds. The preparation and execution of nominal events also occupied far more time than expected. Due to late changes in the LCROSS launch date, Deep Space Network contacts were at highly variable times of day, and at variable intervals. Based on results from earlier science events, the operational profiles of later payload calibrations were modified, requiring extra team effort to accommodate. Furthermore, efforts to remove ice from the Centaur upper stage were less effective than expected, prompting the team to design and execute two additional maneuvers for that purpose. On the second Earth revolution, a substantial spacecraft anomaly caused the MOS to divert from nominal operations for two weeks to save remaining propellant and to guard against similar future problems. On emergence from this particularly taxing recovery period, the team progressed steadily towards a more sustainable operational cadence. The final weeks of the mission interleaved final rehearsals for Centaur separation and impact with nominal operations, and increased the frequency of trajectory maneuvering in preparation for impact. Despite these challenges, the MOS performed with very few operational errors, none of which had measurable negative influence on mission outcome.

Impact Phase was executed nearly flawlessly. The MOS operated for roughly TBD hours continuously, and performed two full operational cycles including the execution of Centaur separation and lunar impact. There were some voice loop communications problems in the final minutes of flight, attributed to shortcomings in training for real-time instrument commanding. These had some effect on data collected at the time of the Centaur impact, but with a negligible influence on overall science goals. LCROSS met all of its mission objectives.

\section{Conclusions}

What were the keys to the success of the LCROSS flight team? Perhaps most importantly, LCROSS benefitted tremendously from a pervasive spirit of cooperation and trust that crossed organizational boundaries. This improved communications at all levels, spawned additional collaborations, and caused people to devote extra time throughout the project to ensure mission success. From the MOS development perspective, securing full-time access to the LCROSS simulator was critically important. It allowed the team to gain early, risk-free experience in the operation 
of LCROSS, and provided the team with a realistic test platform in creating the hundreds of command products to support various mission events. The simulator was also the foundation for its series of MOS tests and rehearsals.

Not surprisingly, frequent, repeated testing of the team under realistic conditions was invaluable. These tests exposed weaknesses throughout MOS development, enabling the team to perfect its equipment, processes, procedures, and staffing schedules before launch. By launch, the team had "flown" each major event multiple times, and this significantly improved team confidence in flight. As an unintended consequence, MOS simulator-based tests were partially responsible for exposing two significant spacecraft bugs prior to launch (subsequently corrected). MOS testing (with MOS-developed flight command sequences) provided a level of realism that could not easily be achieved in standard spacecraft system-level verification tests.

Planning and execution of the flight was not perfect. The MOS was worked very hard for the greater part of the mission. A very challenging development phase, with accumulation of vast overtime hours, continued right up until launch. The flight was busier and the schedule more irregular than anticipated, contributing further to team fatigue. For the most part, the team was getting sufficient sleep to avoid human error, but time off to tend to personal matters was uncommon. Mainly due to lack of time, the team did not train a full set of backups for critical operational positions. Fortunately, the team did not falter on attendance or performance due to illness or accident.

Distributed operations were largely successful. The MOS made substantial improvements to communications prior to launch, and operators from all disciplines made strong contributions to the team, despite being remotely situated. However, communications and situational awareness could have improved even more. Some basic problems (e.g. poor room acoustics) hampered communications between ARC and NG ROC's. Furthermore, the NG ROCs were tasked with partially conflicting roles, exacerbating the communications problems: one to support closely-coordinated procedure execution as extensions of the MOCR (demanding quiet, focus, and attentive voice loop participation), and the other to provide assessments of spacecraft performance (benefitting from offline group discussion). Recognizing these conflicts, we revised ROC protocols mid-flight, with noticeable improvement.

As discussed throughout the paper, staff members that were dually-tasked as MOS operators and GDS developers were unable to fully satisfy the demands of both roles in flight. High-priority MOS tasks consumed most operators' time. Without a sufficient break in the busy flight schedule to perform testing, performing a formal global GDS deployment was seen as too risky. Instead, critical fixes were introduced on isolated workstations.

Despite its success, it is debatable whether the LCROSS approach is an appropriate model for sustainable future, low-budget, high-risk operations. LCROSS had the advantage of being an extremely exciting mission with low initial expectations. The MOS successfully transformed into a highly-capable operations team, but this could not have happened without the extraordinary hours the team contributed from the start of development through impact.

\section{Acknowledgments}

\section{References}

${ }^{1}$ A. Binder, “Lunar Prospector: Overview”, Science, Vol. 281, No. 5382, 4 September 1998, pp. 1475-1476.

${ }^{2}$ W. Borucki, et al., "Kepler Mission: Design, Expected Results, Opportunities to Participate”, A Decade of Extrasolar Planets Around Normal Stars, edited by M. Livio, K. Sahu, and J. Valenti, Space Telescope Science Institute Symposium Series, No. 19, Cambridge University Press, 2005, pp. 36-49.

${ }^{3}$ P. Tompkins, et al., "Flight Operations for the LCROSS Lunar Impactor Mission”, AIAA Space Ops 2010 Conference, Vol. TBD, American Institute of Aeronautics and Astronautics, Washington, DC, 2010, pp. TBD-TBD. 